\title{
Integration of multi-physics analysis into the cabin design process using virtual reality
}

\author{
Christian Hesse* and Jan-Niclas Walther* and Pia Allebrodt* \\ German Aerospace Center, Hamburg, Germany, 21129 \\ Martin Wandel ${ }^{\dagger}$ \\ Airbus Operations GmbH, Hamburg, Germany, 21129
}

\begin{abstract}
The design of an aircraft cabin and especially its disciplinary evaluation is a highly specialized process in the product development of an aircraft. Low-fidelity assessment of a cabin design is not commonly possible as there are many interactions with other disciplines and very detailed inputs are usually necessary in a particular form and format. In order to enable such high-fidelity evaluation processes for complex cabin systems in the early design stages of the cabin, the use of the Common Parametric Aircraft Configuration Schema (CPACS) is proposed in this paper for ensuring digital consistency among different disciplinary tools. Initially developed for the overall aircraft design, CPACS has been extended with a wide variety of definitions for the structural and cabin design. These support the efficient data exchange between the involved disciplines in a model-based design approach. This process is exemplified here by deriving different multi-fidelity and multi-physics simulation models from a central CPACS dataset. For multi-fidelity analysis, finite element models as well as statistical energy analysis models for the vibro-acoustic evaluation are demonstrated. In order to illustrate multi-physics capabilities, static structural and thermal variants for the finite element models are derived as well. A main advantage of using consistent data modelling is the fact, that all results from the different analyses can be fed back to a central visualisation platform. This enables the intuitive and collaborative exploration of the design as well as the results from the heterogeneous disciplines with all involved partners. The visualisation model is also derived from the central CPACS dataset and realised in this study in a virtual reality environment using the Unity game engine.
\end{abstract}

\section{Introduction}

A key challenge for the cabin design in future aircraft generations is predicted to be the mitigation of cabin noise [1, 2]. The reduction of interior noise on the other hand necessitates reliable methods for the prediction of noise sources, the propagation as well as the transmission through the fuselage structure; this information needs to be known as early as possible in the design process in order to increase the cost efficiency of the design. Especially in cabin acoustics, the different disciplines work with largely heterogenic data. This is further complicated by the need to use different models and fidelity-levels for varying frequency domains. Also, the strongly-coupled interaction with other disciplines (and physics domains) like thermal insulation and cabin pressurization are still not fully understood.

A comprehensive overview of vibroacoustic simulation approaches in the aeronautical industry is given by Peiffer [3]. The different modelling methods are subdivided by the addressed frequency domain. For low frequencies a deterministic approach like the finite element method (FEM) is utilized. Although the use of finite elements with quadratic shape functions may increase the bandwidth of applicability of FEM [4, 5], it can not cover the entire audible frequency range. Therefore, at ascending frequencies an increasing number of subsystems are replaced by random models by employing the statistical energy analysis (SEA) in a hybrid implementation. The main restriction for a full digital workflow for cabin noise prediction according to [3] lies in the distribution over a large number of software suppliers and the underlying need for using consistent data across different companies and tool landscapes.

With its CPACS (Common Parametric Aircraft Configuration Schema) data format [6], DLR has developed an exchange standard to ensure such use of consistent data spanning multiple disciplines as well as levels of fidelity. Especially the structural aircraft definition features a high level of detail which makes it suitable to generate high-fidelity

\footnotetext{
* Research assistant, Institute of System Architectures in Aeronautics, Cabin and payload systems, Hein-Saß-Weg 22

${ }^{\dagger}$ Expert Interior Noise Prediction and Simulation, Interior Noise, Kreetslag 10
} 
vibroacoustic models. A CPACS dataset for the Acoustic FLight-LAB [7] has been compiled in the currently available version 3.2 by the authors. The aim of this study is to present the generation of simulation models applicable in the lowand high-frequency domain from such a dataset. By comparison with an already validated simulation model [8] the generated model can be verified on a barrel aircraft section. The structural definition in CPACS on the other hand spans the entire aircraft (including the fuselage and wing as well as the wing box structure). The methodology is therefore estimated to scale quite well for model generation on the aircraft level. Together with the recent advances in modelling cabin furnishing components [9] it is therefore a suitable foundation for evaluating interior cabin noise.

An overview of the evaluation scheme used in this study is given in Fig. 1 in the form of an extended design structure matrix (XDSM) [10], where the disciplines are arranged on the main diagonal. In the XDSM the black lines describe the process sequence and the grey lines indicate the flow of data. Here, the input $x_{0}$ denotes the central CPACS file which is identical for each disciplinary analysis in order to ensure consistency. The outputs $y_{i}$ describe the results from the respective disciplinary analysis. E.g. $y_{1}$ represents the thermal distribution in the structural components which is used in a static structural analysis as a load for a linear perturbation analysis. Results $y_{2}$ from the analysis are then taken as a pre stiffening load for a modal analysis of the fuselage structure. All outputs $y_{1} \ldots y_{4}$ are then fed to the VR environment for visualization in a common model. This is achieved by hierarchically structuring each model and result dataset by a common descriptor, namely the universal identifier (UID). The result data can then be mapped to the visualization model on a component-wise basis.

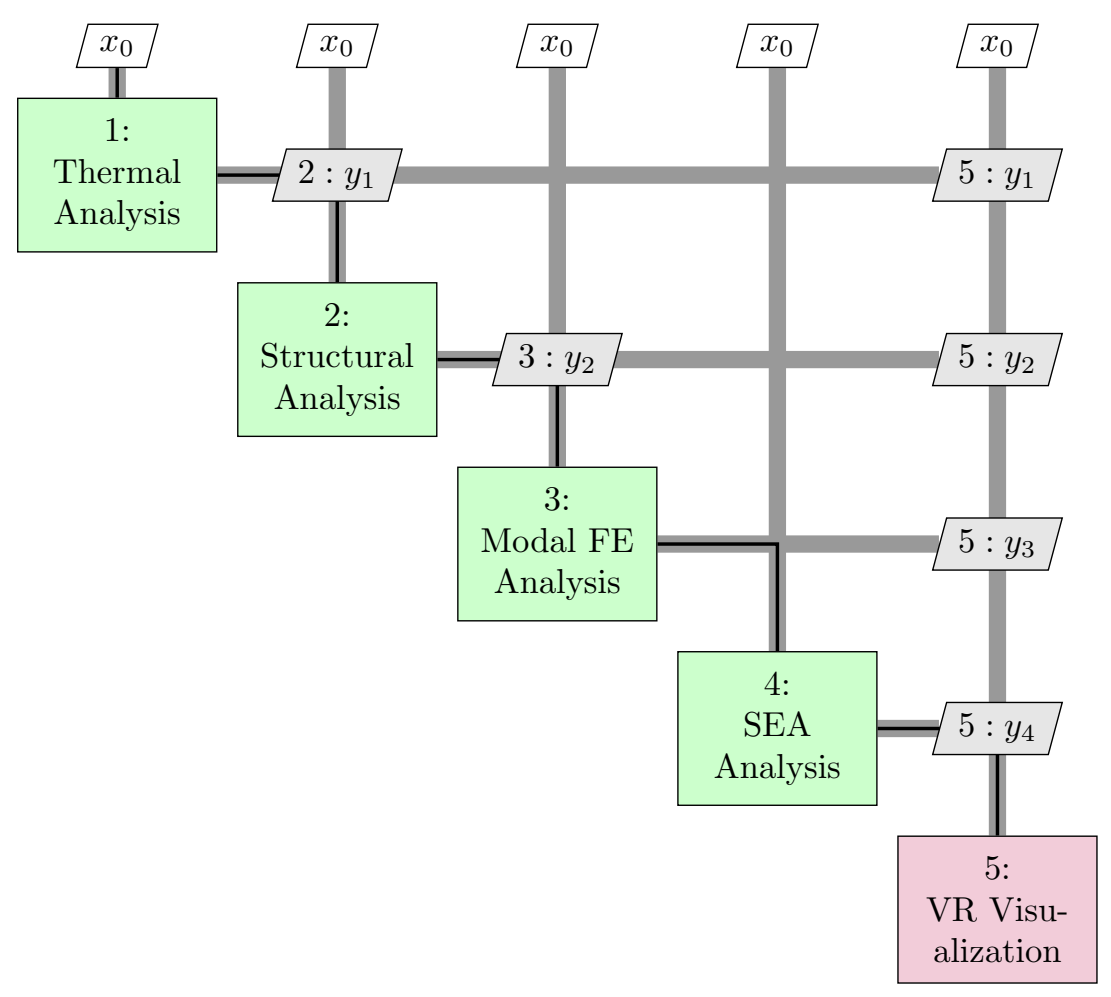

Fig. 1 Extended design structure matrix for multi-disciplinary analysis

The advantages of using consistent data modelling along the disciplines as well as a central evaluation platform are discussed in this paper. Using the interfaces for consistent collaboration and digital continuity from a disciplinary view, contributes to the ultimate goal of a digital thread as envisioned by academia and aerospace industry. Sec. II describes the multi-physics modelling using interfaces to the central data format. The results of the disciplines are shared between one another and are then integrated into a virtual reality environment using the Unity game engine. This bears the potential of an independent visualization platform, which enables a comprehensive and integrated evaluation of the cabin design. The integration of the multi-disciplinary results into a virtual reality environment is further detailed in Sec. III and different displacement-mapping methods are discussed. Sec.IV concludes the study and gives a brief outlook to further examinations. 


\section{Multi-fidelity and multi-physics modelling from CPACS}

This section introduces the multi-fidelity and multi-physics modelling scheme from a central data format, namely CPACS. A CPACS dataset is usually initiated in the overall aircraft design process where an outer fuselage shape is defined. It is then augmented in a structure and cabin design process [9]; an example dataset for the Acoustic FLIGHT-LAB is used in this study. This test structure to be analysed is therefore first introduced in Sec.II.A. The FE and SEA model generation processes are presented in Sec. II.B and II.C respectively. Also, results from both models are given to demonstrate the consistency between them. Finally, selected results from multi-physics analysis and their interactions are described in Sec.II.D

\section{A. Acoustic Flight-LAB}

The analysed test structure is the Acoustic Flight-LAB shown in Figure2 2 inside its testing environment, located at the Center of Applied Aeronautical Research (ZAL) in Hamburg. The fuselage section of $8.5 \mathrm{~m}$ length has a diameter of approximately $4 \mathrm{~m}$ and is based on a short-range narrow-body passenger aircraft made mostly from aluminum alloy. The fuselage barrel has a floor for the passenger cabin as well as for the cargo bay. The passenger floor is mounted to the fuselage at every frame position via a crossbeam and two struts. The skin is stiffened with 87 stringers in circumferential and 17 frames in longitudinal direction. It therefore consists of 1392 skin fields and has an approximate mass of $1500 \mathrm{~kg}$.

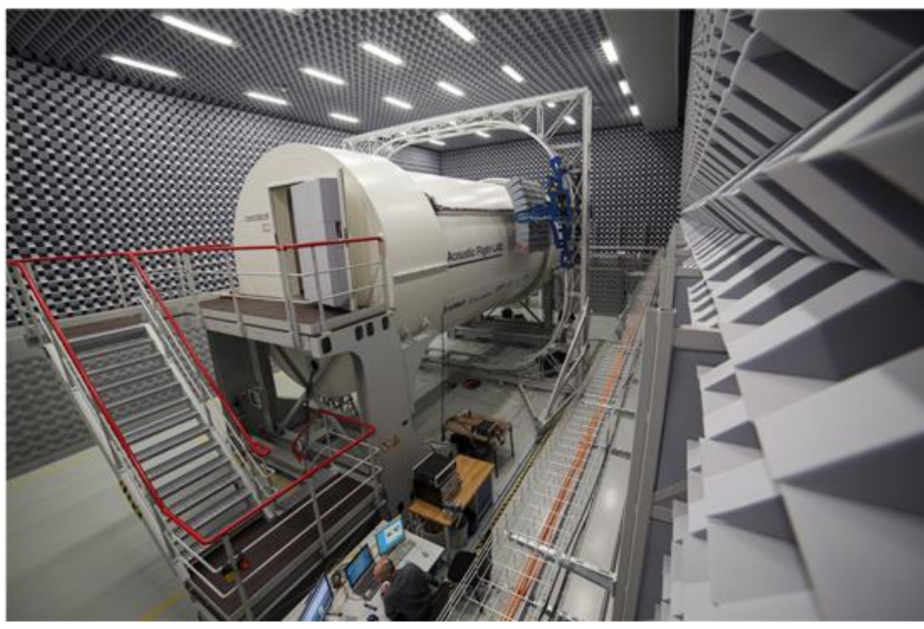

Fig. 2 Acoustic Flight-LAB in the testing environment [8]

The fuselage section is mounted between two portals and supported by four very soft air springs at the end crossbeams, which decouple the structure from the support. Both endings of the structure are isolated with acoustic absorbers. The vibro-acoustic finite element (FE) model of the primary structure provided by Airbus Operations GmbH is used as a reference throughout this paper. The validation of the FE model is described in detail by Wandel et al. [8] using high-resolution vibration measurements. The classification of the low-, mid- and high-frequency range of the test structure is described in [11] on the basis of a spatial Fourier decomposition of the operational deflection shapes. Up to $\approx 160 \mathrm{~Hz}$ the structural vibration exhibits a mostly global behaviour. Beyond this frequency an increasing number of local modes of the skin fields and stiffeners contribute to the structural vibration in addition to the global modes. Above $\approx 300 \mathrm{~Hz}$ the structural vibration is completely random in nature and the vibration response from skin fields and stiffeners are mostly uncorrelated. The entire Acoustic FLIGHT-LAB test structure and all its structural components are currently describable in the current CPACS version 3.2. For a detailed description and the rationale behind the definition the reader is referred to [12]. The structure has been compiled into a CPACS dataset which is used in this study as a common baseline for the finite element model generation. These simulation models can be used in multiple disciplines (structural, thermal and acoustics). The model generation is described in the following section.

\section{B. Finite element model generation}

The finite element model generation from the previously defined CPACS dataset is detailed in this section. The different models are subsequently used for structural, thermal and vibro-acoustic analysis. Multiple geometry libraries 
are available for the creation of nodes and element connectivities from CPACS data and two libraries are supported in this work in particular. The official companion library TiGL [13] can be used as geometry pre-processor for the FE model generation through their MATLAB bindings. Another available library is the yet another CPACS geometry library (yacgl) [14] which is especially applicable where detailed structural geometries like e.g. extruded beam profiles are needed. Each finite element model is created for ANsys as a block-formatted cdb-file.

Creation of the structural components is subdivided into different structural parts. Firstly, each skin field is created with its respective frame and stringer connections attached to its edges. The skin fields are modelled with SHell181 elements for structural and SHell131 elements for thermal analysis. When using TiGL, the function tiglFuselageGetPointAngleTranslated is used to get multiple points per skin panel evenly spaced on the fuselage loft. The stiffeners and floor structure beams can either be modelled as beams of type BЕAм188 or they are extruded as SHELl181 elements. For the second option, the yacgl is used for the creation of the extruded profiles. In a next step, the interior structures containing crossbeams, crossbeam struts as well as longitudinal floor beams of the passenger and cargo floor are built up accordingly. The supporting structures are then connected to the revolving frames. Lastly, the floor panel elements are assembled and connected to the longitudinal floor beams.

After these steps, the individually meshed structural component are uncoupled from each other and no connection is available. A contact definition between different structural components, e.g. skin fields and stringers or frames, therefore needs to be established. This is realized by utilizing an idealized riveting model as well as surface contacts. For the definition of surface-to-surface contact a combination of TARGE170 and CONTA174 surface elements is used. Also, riveting connections are modelled using surface-to-node contact on each side and connecting both nodes with bushing elements of type Сомві250.

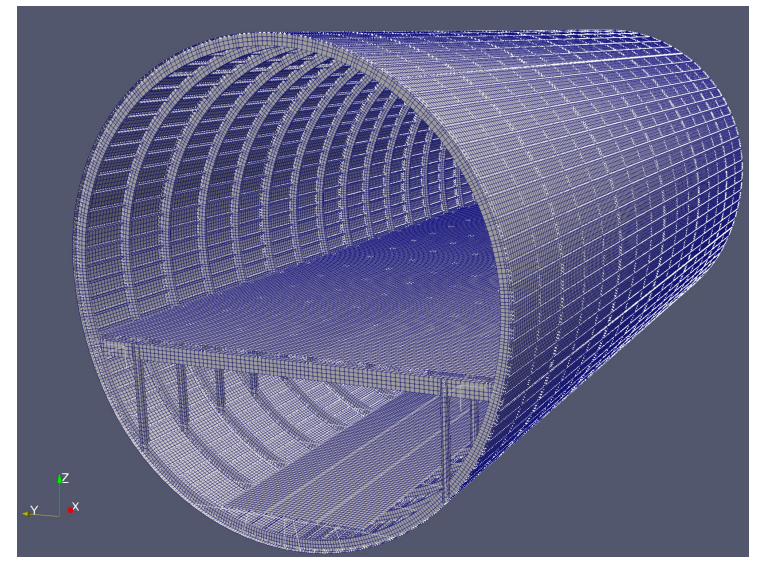

(a)

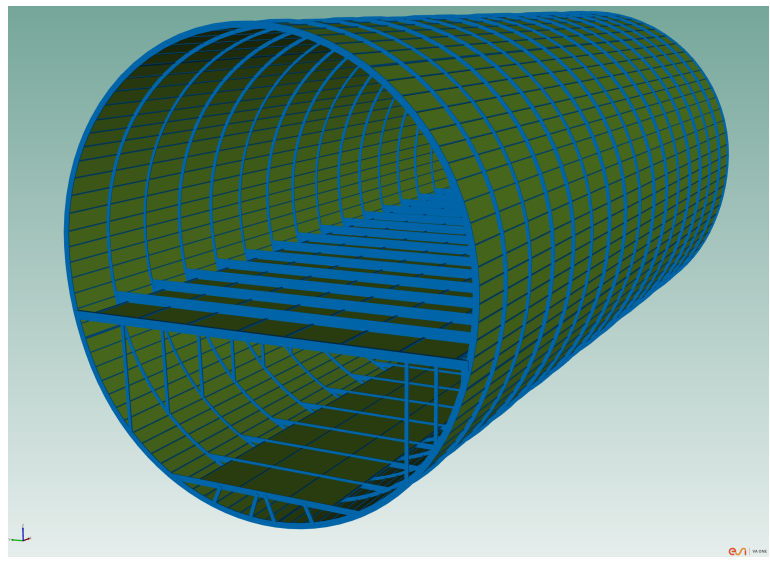

(b)

Fig. 3 Exemplary FE (a) and SEA (b) models generated from CPACS dataset

An exemplary FE model of the fuselage structure for vibro-acoustic analysis is shown in Fig. 3a Here, a minimal target length for each element edge of $0.04 \mathrm{~m}$ is assumed. This way, a total of 7 elements per bending wavelength is satisfied up until a frequency of $160 \mathrm{~Hz}$ for the fuselage skin. A discussion of the usual criterion of more than 6 elements per wavelength can be found in [15]. Although in a static analysis a much coarser grid is sufficient, the identical model is used here in order to ensure a rapid mapping process from one model to the other. Alternatively, if differently sized models are used, a mapping process as described in Sec. III.C is still applicable. For subsequent thermal analysis, only the fuselage skin is modelled. All other structural components are assumed to exhibit their reference temperatures, i.e. room temperature. The following section describes an analogue model generation process for the statistical energy analysis.

\section{Model generation for statistical energy analysis}

The statistical energy analysis is a method for the simulation of vibro-acoustic systems in the high-frequency regime. In this study, the analyses are being conducted using the software VA One [16]. This software reads an xml file format for the model input which is build from the CPACS dataset. Like for the finite element model generation, the TiGL library is used for the geometrical pre-processing. Contrary to the finite element models, all stiffener elements are 
represented here by beam elements. The stiffener profile properties are calculated using the Python module Prometheus (Profile Mesh Thickness Elementation Utility Script) [17]. The material and composite properties are read from CPACS and converted to the new xml file using the TiXI library [13].

In SEA analysis the subdivision into weakly coupled subsystems is of great importance. Also, the modal density of the subsystems plays an important role for the valid representation of the energy content. Therefore, the subdivision of the SEA model shown in Fig. 3b is briefly explained in this section. Each frame is represented by one circular beam element; each stringer is modelled by one continuous beam element respectively. The stringer-frame-assembly is connected to each other by point-junctions and the embedded skin panels are modelled as singly curved shells and connected via line-junctions. As each skin field is fairly small, a sufficient modal density ( $>3$ per third octave band) can be expected only for frequencies upward of $800 \mathrm{~Hz}$. An alternative way to define the panels is the subdivision by the skin segments, which span multiple skin fields and are defined by start and end stringers as well as frames [12]. For this, the ribbed panel property must be used in SEA analysis in order to include the stiffness of frames and stringers on the skin segment. The crossbeam stiffeners are attached via point junctions to the frames and the longitudinal floor beams are connected to the crossbeams in the same way. The struts for the passenger and cargo crossbeams are attached to the crossbeams on the upper end and to the frames on the lower. Here, also point junctions and the respective connections are used. Each floor panel is connected to its associated longitudinal floor beams via point junctions where it is usually bolted on.

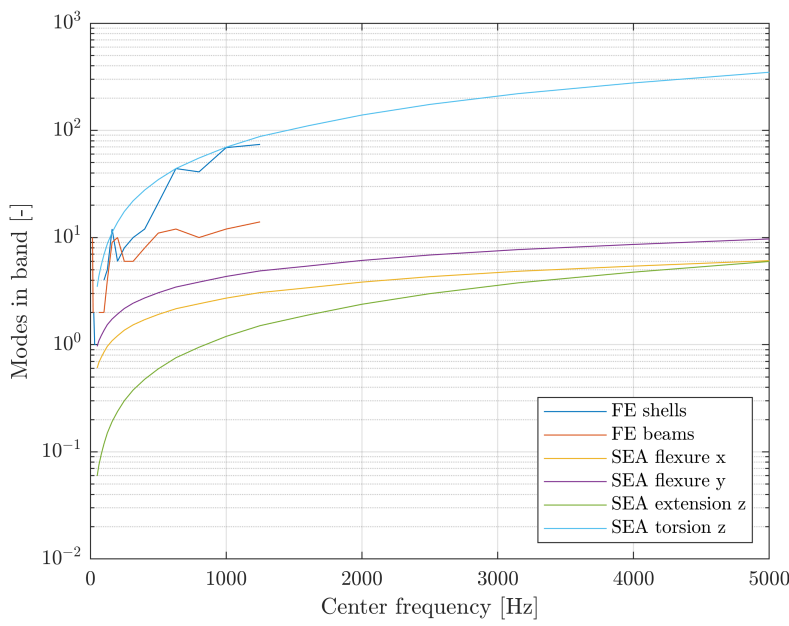

(a) Frame

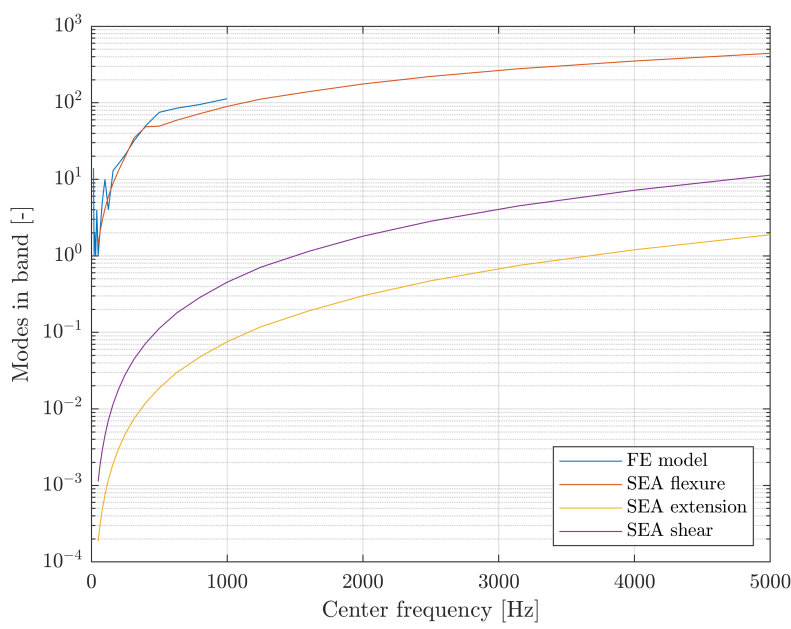

(c) Skin segment

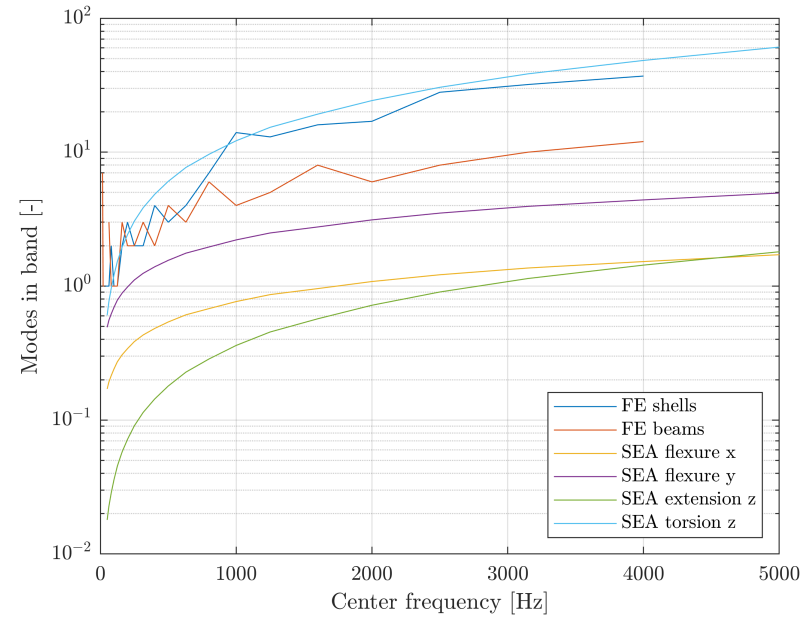

(b) Pax crossbeam

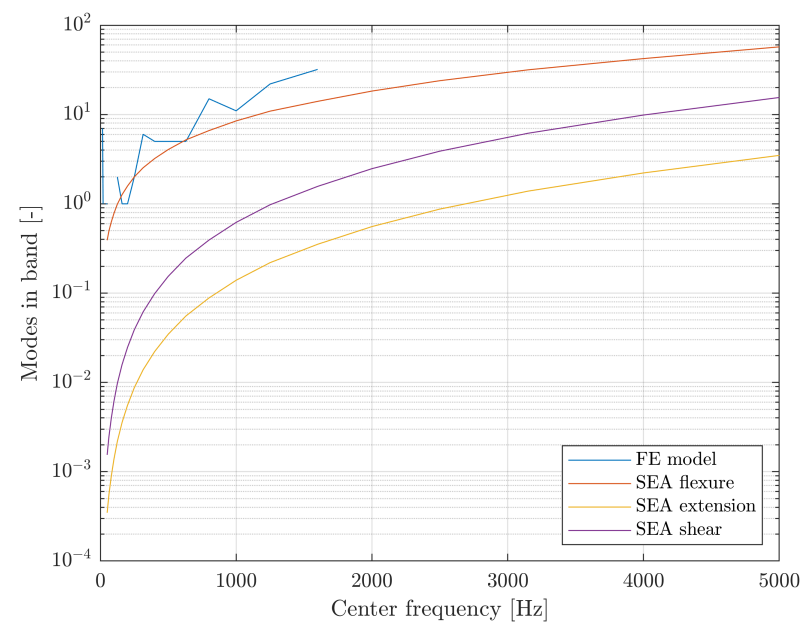

(d) Floor panel

Fig. 4 Modes in third octave band comparisons for different structural components between FE and SEA models 
In order to demonstrate the consistency between the SEA and FE model, Fig 4 compares the number of modes in third octave bands for selected subsystems. Here, two beam type and two shell type subsystems have been selected. The respective FE models were each meshed sufficiently fine, in order to address a high enough frequency range. For the beam type subsystem (frame and passenger crossbeam) both beam and extruded shell models as described in Sec.II.B where examined. It can be observed, that the FE beam definitions shown in Figs. $4 \mathrm{a}$ and $4 \mathrm{~b}$ are too stiff compared to the SEA formulation and the modal densities are too low as a result. Both extruded shell models compare reasonably well to the the SEA model which is dominated by torsional modes in the entire frequency range. The results for the skin segment (which is modelled in SEA analyses as a singly curved shell spanning two frame and 14 stringer bays) and the floor panel are depicted in Fig. $4 \mathrm{c}$ and $4 \mathrm{~d}$. Both results are fairly similar between FE and SEA formulations. Although both methods address completely different frequency ranges, this suggests a high degree of consistency for the structural components between both models. A more detailed comparison between the analysis results, also with experimental measurements detailed e.g. in [18], will be done in further studies.

\section{Multi-physics analysis}

In this section the generated finite element models for structural, thermal and vibro-acoustic analysis are used to study the interactions of the different disciplines. Due to them being generated from the same CPACS dataset, they are completely consistent to one another and the results from one discipline can be efficiently mapped to another discipline. Consequently, the calculated pretension from a thermal analysis can be used in the vibro-acoustic analysis. In the flight state of an aircraft this originates from the fact, that the outside air is much colder than the inside air. The resulting temperature gradient and the expansion or contraction of the material (which strongly depends on the thermal insulation characteristics) therefore leads to a pretension of the structure which also affects the structural vibration modes. Also the loads from a pressurized cabin analysis can be applied in order to study the effect on the mode shapes and eigenfrequencies of the structure. A brief overview of the results is given in this section.

A total of three load cases $\mathbb{L}_{1} \ldots \mathbb{L}_{3}$ and a reference analysis $\mathbb{R}$ is considered in this section. For the reference analysis $\mathbb{R}$ a modal analysis is conducted without exterior loads. In load case $\mathbb{L}_{1}$ a thermal analysis is first conducted and the resulting load is applied to the structure using the linear perturbation process [19]. The resulting eigenfrequencies can then be compared to the unloaded case. Load case $\mathbb{L}_{2}$ replaces the thermal load with a cabin pressure load from a static structural analysis. In load case $\mathbb{L}_{3}$ both loads are combined.

A comparison of the reference analysis $\mathbb{R}$ for different FE models is shown in Fig 5 . Here, the eigenfrequencies (above the rigid body modes) for an increasing target element length in the model generation process are compared. With decreasing element length, continuously decreasing eigenfrequencies are evident for all vibration modes. As a plausibility check, the resulting eigenfrequencies from the NASTRAN reference model [8] are plotted as well. It should be mentioned here, that the aim of this study is not the recreation of an already existing simulation model. Rather, it is emphasized that the CPACS data format delivers enough fidelity to generate different simulation models which address certain modelling aspects, fidelity levels as well as frequency-domains. The reference model contains a lot of specialised element parameters which are impossible to obtain in early design stages. E.g. a lot of different rivet stiffnesses are included for the contact definitions of different structural components. These were identified by the comparison with experimental measurements [18]. In the current model generation process, one generic riveting stiffness is utilized for each contact. Also, the complex three-dimensional intersections of components, e.g. extruded crossbeams with fuselage skin, have been somewhat simplified here. The resulting eigenfrequencies nonetheless compare reasonably well to the reference model in the low-frequency regime.

A preceding thermal analysis is conducted for load case $\mathbb{L}_{1}$. For the comparative nature of the study, a use-case for thermal analysis is selected here, where the main insulation is omitted for simplicity and only the structural skin of the fuselage is considered. The fuselage skin (consisting of Shell131 elements) allows the definition of top and bottom temperature. In steady flight condition, the outer wall temperature $T_{w}$ can be approximated by the surrounding air temperature $T_{\infty}$ and the aerodynamic heating from the boundary layer surrounding the aircraft skin [20] as

$$
T_{w}=T_{\infty}\left[1+r\left(\frac{\kappa-1}{2}\right) M_{\infty}\right] .
$$

Here, $\kappa$ describes the adiabatic exponent and $r=\sqrt[3]{N_{P r}}$ a recovery factor with the Prandtl number $N_{P r}$. As the inner temperature, room temperature (corresponding to the materials reference temperature) is assumed.

The cabin pressurisation and the resulting pressure differential between the inside and outside is to be considered in load case $\mathbb{L}_{2}$. For this, a surface load is introduced on the aircraft skin elements for the static analysis. A pressure 


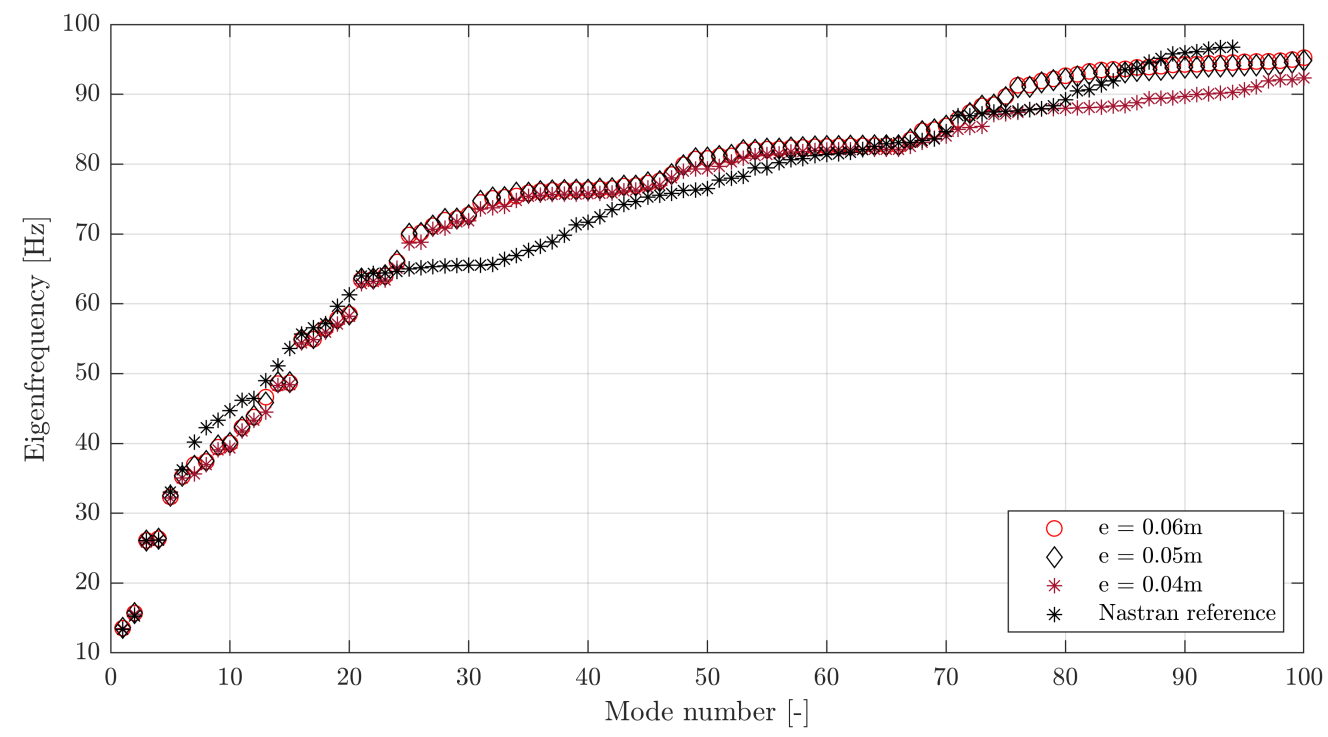

Fig. 5 Comparison of the fuselage structure eigenfrequencies for reference analysis $\mathbb{R}$ with the Nastran reference model

differential of $\approx 48.2 \mathrm{kPa}$ corresponding to a flight level of $10000 \mathrm{~m}$ and a cabin altitude of $2500 \mathrm{~m}$ has been used in this study. The structural loads are then transferred to the modal analysis via linear perturbation just as in the thermal load case.

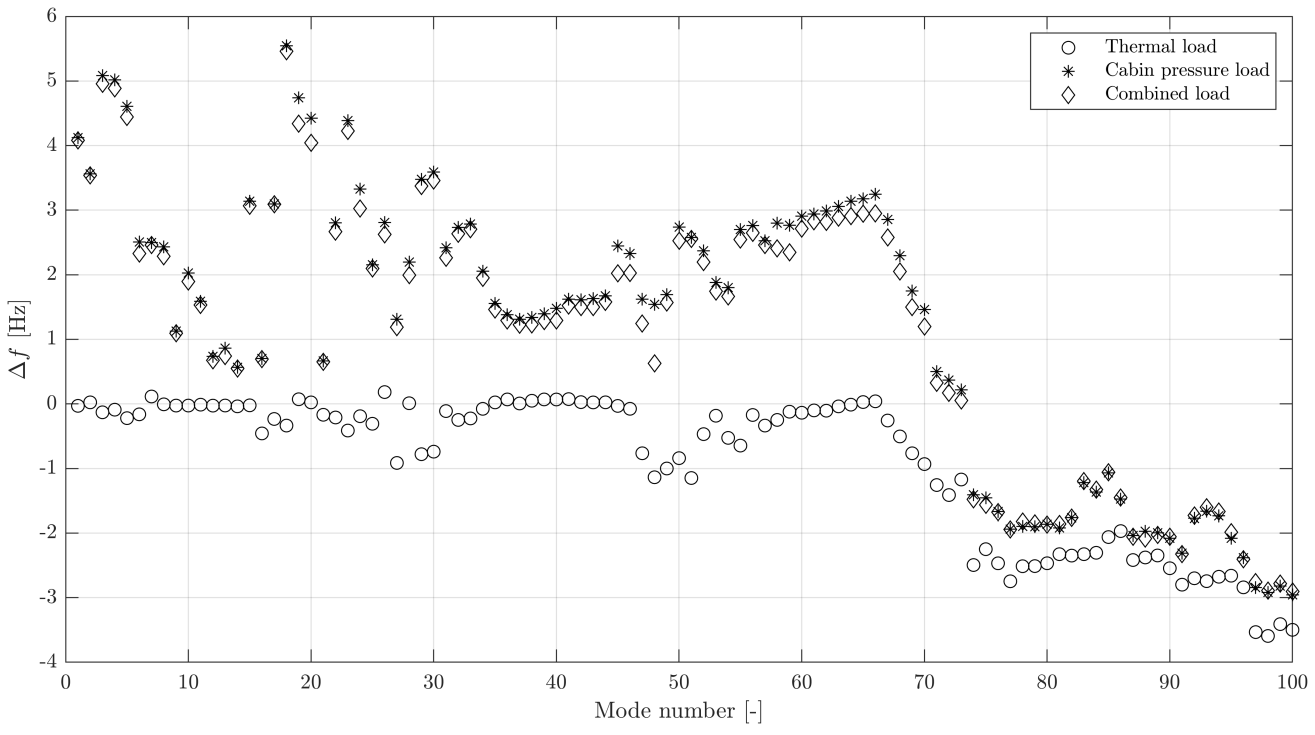

Fig. 6 Influence of thermal and structural loads on the eigenfrequencies of the fuselage structure eigenfrequencies

An overview of the influence of additional loads on the structural eigenfrequencies is given in Fig.6 Here, the frequency difference $\Delta f_{m}=f_{m, \mathbb{L}_{i}}-f_{m, \mathbb{R}}$ is plotted for the first 100 modes above the rigid body modes. It should be mentioned, that no correlation criterion (e.g. MAC: modal assurance criterion) has been used for the ordering of the eigenfrequencies depending on the mode shapes. Thus, the compared frequency pairs must not necessarily correspond to the identical modes. The difference merely indicates the dependency of the structural eigenfrequencies on these external loads. The thermal loads have only a minor impact on the low-frequency global modes. However, the influence increases for the higher mode orders beginning from $\approx 80 \mathrm{~Hz}$. For the cabin pressure loads a significant impact is 
observable from the lowest order modes. In the combined load case analysis it becomes apparent, that the influence from the pressurisation load case dominates the analysis as the results are very similar for all modes considered.

The current section was concerned with using consistent modelling across multi-fidelity and multi-physics analysis. For the model generation in each discipline, the central data format CPACS has been used. A first CPACS dataset is usually initiated in overall aircraft design and then passed to a structure and cabin design process, described e.g. in [9]. The following section brings the different results from the analysis models back together using a central visualization model which is realized in a virtual reality environment.

\section{Virtual reality integration}

This section discusses the integration of the disciplinary analysis processes in a virtual reality environment for collaborative and rapid evaluation. First, the virtual reality environment and its requirements regarding the result inputs are reviewed in Sec. III.A. For use in a virtual reality environment the structural and cabin geometry components are assembled from the identical CPACS dataset used for the model generation. The geometry components are exported as triangulated or meshed surfaces where the original hierarchy is preserved. This allows for the consistent mapping of analysis data to the VR model. For this, a mesh-free mapping scheme, as described in Sec. III.B is used. Sec.III.C and Sec.III.D discuss different methods to include disciplinary results in a virtual reality environment.

\section{A. Virtual reality environment}

The central visualisation environment is realized in the Unity game engine as it is flexible and customizable to the users needs. The geometry model is created directly in the cabin and structure design process [9, 14]. The uIDs of all structural and cabin components from the CPACS dataset are retained for the mapping process and also for the visualisation. Each geometric component is exported from the Python process in a separate file, where the hierarchy is described through an xml-file. The $s t l, o b j, f b x$ and $g l t f$ file formats are currently supported for the definition and transfer of meshed geometries. The result of this process is depicted in Fig.77 which shows the hierarchical subsystem division in the Hierarchy window of Unity as well as the combined visualisation model assembled from the individual meshes.

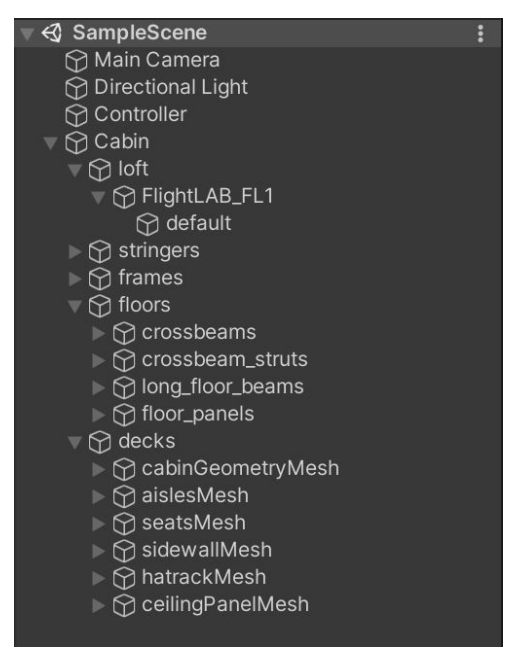

(a)

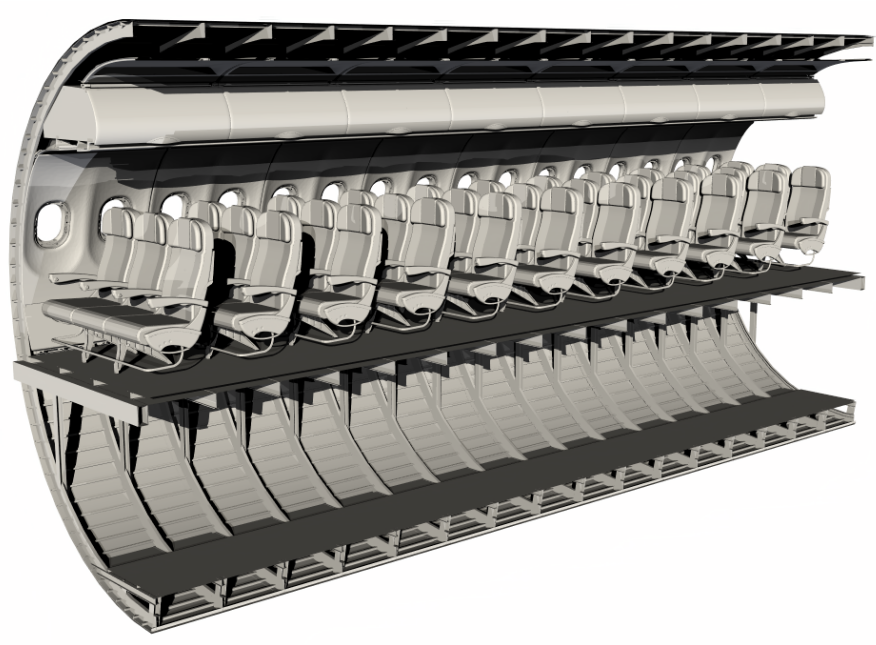

(b)

Fig. 7 Hierarchical subsystem division in the Unity engine and combined visualisation model

The aim of this study is the integration of the disciplinary results in this central visualisation model. All results from ANSYs are written to the $v t u$ file format using the pyansys package [21]. Results from the SEA analysis can be written to a $h d f 5$ file and mapped according to the subsystem definition. This latter process has not been implemented yet. Since in the FE model generation process the element sets were exported and named after the CPACS uIDs, the exported results can be allocated back to the structural components. The same is true for the SEA analysis, where the subsystems are named by the $u I D s$. These results are to be visualized in the virtual reality; for a proof-of-concept this paper focuses on visualising the modal displacements in the form of deformations as well as textures. For a visualisation of the 
nodal deformations, the $3 \mathrm{~d}$-displacements are mapped to the nodal coordinates in the visualisation model as detailed in Sec. III.C. Alternatively, for the textures option, the magnitude results are mapped to the local surface coordinates of the geometry models. This process is further explained in Sec.III.D.

\section{B. Mesh-free Mapping}

Mesh-free mapping schemes are well-established in fields such as aeroelasticity where data must be exchanged between non-matching analysis meshes. Fundamentally, the idea is to transfer point data, such as displacements, from a set of source points to a set of target points by way of a mapping matrix $\mathbf{H}$ using

$$
\mathbf{u}_{\text {target }}=\mathbf{H} \cdot \mathbf{u}_{\text {source }} \text {. }
$$

Different methods have been proposed to compute the matrix $\mathbf{H}$, such as Radial Basis Functions (RBF) by Beckert and Wendland [22] and Moving Least Squares (MLS) by Quaranta et al. [23], which are applied in the following using the implementation presented in [24]. It follows from Eq. 22, that $\mathbf{H}$ is a $n_{x, \text { target }} \times n_{x, \text { source }}$ matrix. Therefore, the memory footprint of the mapping operation is highly dependent on the meshes involved and can become a limiting factor for very large meshes. Both methods mentioned share the common goal to reduce the memory requirements by expressing $\mathbf{H}$ as a sparse matrix. This is achieved by introducing compactly supported basis functions $\phi_{\Gamma_{1}, \Gamma_{2}}$, which restrict the influence of a point to its immediate vicinity defined by a support radius.

In the RBF algorithm, the mapping matrix is computed using a global approach. Despite the compactly supported basis functions, it requires the computation of the inverse of a sparse $n_{x \text {,source }} \times n_{x \text {, source }}$ matrix, which returns a non-sparse matrix of the same size. Therefore, the application of the algorithm remains restricted by the size of the source mesh making it especially well suited for mapping data from coarse to fine meshes. On the other hand, the RBF algorithm is an interpolationg algorithm, which promises more accurate results.

In the MLS algorithm, the localized approach is expanded by breaking the problem down into a series of local problems, which are solved independently using a least-squares approximation. This yields one row of the mapping matrix $\mathbf{H}$ for each point in the evaluation set $\mathbf{x}_{\text {target }}$. The benefit of this method is that no solutions or inversions of a large system of equations is necessary, making it even more memory efficient than the RBF algorithm. However, it can only provide an approximation, as opposed to an interpolation, as provided by the RBF algorithm. An interpolating version of the MLS algortihm has also been proposed by Krishnamurthy [25], which, however, compromises the memory benefits again.

\section{Node-based displacement mapping}

At first, a node-based displacement mapping approach analogous to the displacement mapping in fluid-structure interaction is considered. In this case, the displacement values at the FE analysis mesh nodes in Fig. 3a are mapped onto the VR mesh nodes in Fig.7b. In order to achieve good performance during rendering, the VR mesh is usually very coarse, with flat faces often represented by no more than 2 triangles. This means that, contrary to common applications, the source mesh offers a much better resolution of the domain, than the target mesh. Therefore, it may not be capable of reproducing certain deformation shapes. This can be alleviated by re-meshing the underlying geometry of the VR mesh using a meshing tool such as Gmsh [26] with a constraint on triangle size.

Due to the size of the source mesh, the MLS algorithm is applied for the mapping using a Wendland C2 basis function and a support radius $r_{\text {support }}=1 \mathrm{~m}$. The result for one solution case is given in Fig.8, which depicts a deformation mode shape of the source and target meshes. Here it becomes apparent, that the approach is robust enough to handle structural dissimilarities, e.g. the window cut-outs in the target mesh. Fig. 8 also illustrates a major advantage of the node-based approach, namely the possibility to apply the mapped deformations to the target mesh without any further processing. The results show an acceptable resemblance, due to the increased mesh quality of the target mesh. Choosing a higher mesh resolution will improve the quality of the mapping further, at the cost of visualization performance in real-time applications.

Closer examination reveals inaccuracies, which can be traced due to the approximating nature of the MLS algorithm. These occur at displacement maxima and especially at the boundaries of the meshes; however, they are not visible in Fig.8. Consequently, the node-based displacement mapping approach is not unconditionally suitable to provide an accurate transfer of physical data to the VR model. Also, the implementation in the Unity engine is not directly supported and requires a custom solution. However, it can be useful to provide a visual impression of the deformations in a VR environment. 


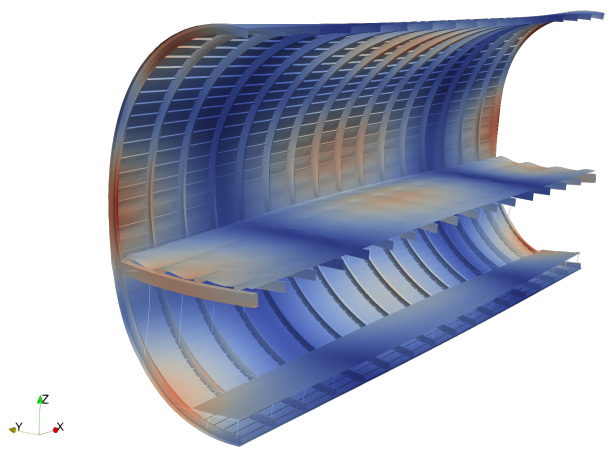

(a)

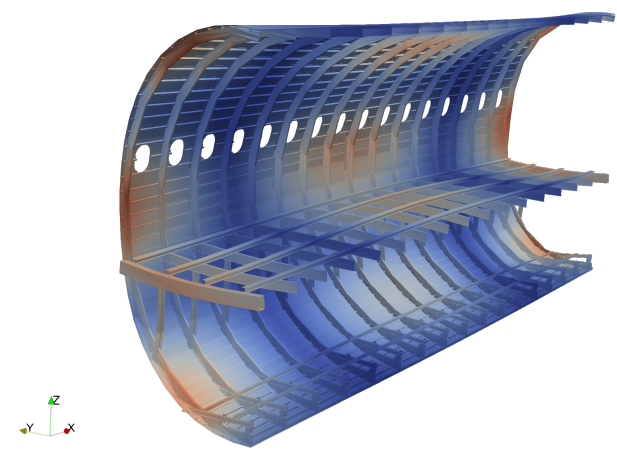

(b)

Fig. 8 Visualization of the deformed source mesh (a) and the mapping results on the target mesh (b)

\section{Texture-based displacement mapping}

Due to the shortcomings of the node-based approach in the previous section, a second method is investigated. This time, instead of mapping the displacements from the analysis mesh to the VR mesh, the local surface coordinates $(u v$ coordinates) of the VR mesh are mapped to the analysis mesh. In this way, each node of the analysis mesh is projected to the $u v$ plane of a corresponding VR mesh surface. Consequently, unlike the previous approach, this approach maps data from the coarse mesh to the fine one, allowing application of the RBF algorithm.

Since the faces in the VR mesh each have their own $u v$ space, a partitioned approach is necessary. The individual components in both meshes must be identified, which is feasible using the $u I D$-sets identified by the CPACS components, provided in the analysis mesh.

Taking the skin surface as an example, which is extracted from the analysis model by merging all skin panel sets, the displacements can be mapped to the surface coordinate space to yield the distribution shown in Fig.9a. Again a Wendland C2 basis function with a support radius $r_{\text {support }}=1 \mathrm{~m}$ is used. The distribution can be resampled and written as an image file, which is then applied to the model as a texture. Fig.9b shows the texture applied to the surface loft model in the scene window of the Unity UI.

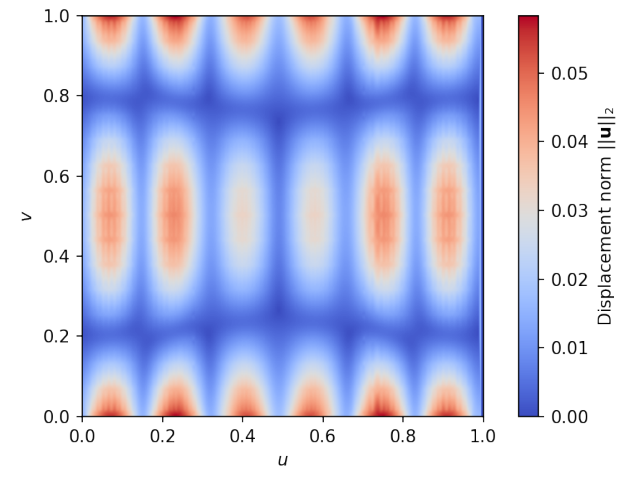

(a)

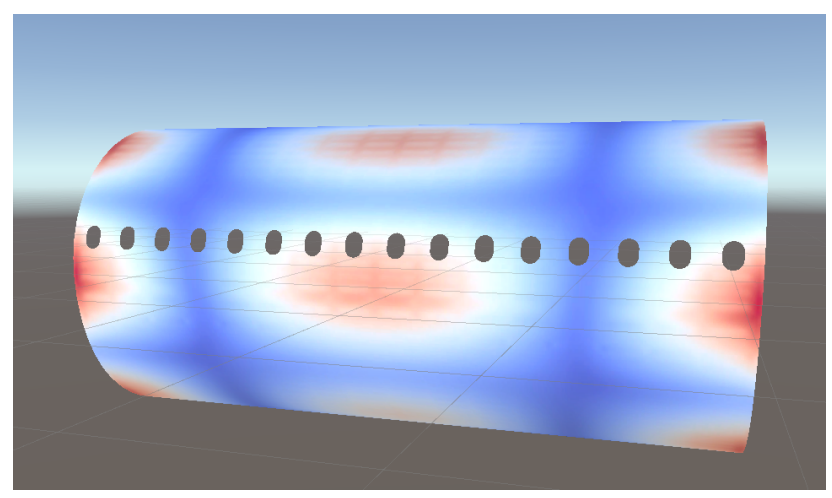

(b)

Fig. 9 Projection of the mesh data to $u v$ space (a) and application as texture in Unity (b)

The results of the texture-based mapping approach look promising, since the mapping is at once more efficient and yields superior results compared to the node-based approach. This is due to the application of the RBF interpolation algorithm. At the same time, the approach appears to be much better suited to provide the displacement data to Unity, which accepts the textures without any further processing. However, this method requires more detailed information on 
the component hierarchy to be available in both meshes. It was shown in this section, that the CPACS interface is a suitable means to provide and ensure this component hierarchy for both node-based and texture-based approaches.

\section{Conclusion}

In this paper, an approach for deriving consistent simulation models from preliminary aircraft design data provided in a CPACS dataset has been demonstrated. Thermal, structural and vibro-acoustic FE models for application in low-frequency cabin acoustics have been generated as well as SEA models for the high-frequency regime. The consistency between the different models has been illustrated by comparing the modes in band between the FE and SEA representations. Also, selected results from a linear perturbation analysis were documented. In the latter analysis, several load cases representing cabin boundary conditions were used to augment a vibro-acoustic FE model for a modal analysis. These results highlight the cross-dependencies between the different disciplines. The integration of these simulation results in a VR environment were discussed and a proof-of-concept was shown for both mapping of 3d displacement data as well as $2 \mathrm{~d}$ textures onto the visualisation models. The mapping of textures was found to be the most promising approach as it is more accurate due to the use of the Radial Basis Functions algorithm.

The workflow has been exemplified using a CPACS dataset representing the Acoustic Flight-LAB structure, a generic fuselage barrel used for vibro-acoustic testing. This CPACS dataset has already been enhanced in order to also include furnishing components from the aircraft cabin, as demonstrated in Fig.77. These additional structural components as well as the thermal and acoustic insulation need to be further incorporated into the model generation processes. On the other hand, the entire aircraft including the pressure bulkheads, wing-fuselage connection regions can also be considered.

It was also demonstrated, that CPACS is suitable as a common denominator for disciplinary model generation as well as result visualisation. The visualisation was limited here to generalized disciplinary results. The inclusion with further disciplines like cabin system design [27] as well as assembly processes [28] in a central VR model is planned for future work. Overall, this will contribute to the DLR's goal of becoming a virtual OEM with the capability of digitally depicting the entire development cycle. Feeding back these disciplinary analysis results to the virtual product will enable a comprehensive and integrated concept evaluation of the cabin within the virtual development process.

\section{Acknowledgments}

The authors would like to express their gratitude to the Esi Group and in particular Dipl.-Ing Achim Ehrhard and Johannes Seidel M.Sc. who generously supplied a test license and support for VA One used for some of the work presented in this paper.

\section{References}

[1] Scheel, H., "Next generation aircraft-A challenge for Interior Acoustics Developments," INTER-NOISE and NOISE-CON Congress and Conference Proceedings, Vol. 253, Institute of Noise Control Engineering, 2016, pp. 4666-4675.

[2] Thomas, C., and Scheel, H., "Kabinenakustik in der Luftfahrtforschung," Deutscher Luft- und Raumfahrtkongress, 4.-6. September 2018, Friedrichshafen, 2018.

[3] Peiffer, A., "Full frequency vibro-acoustic simulation in the aeronautics industry," Proceedings (Keynote) of ISMA 2016 including USD 2016 International Conference on Uncertainty in Structural Dynamics, Leuven, 2016, pp. 1-15.

[4] Langer, S. C., and Blech, C., "Cabin noise prediction using wave-resolving aircraft models," PAMM, Vol. 19, Wiley Online Library, 2019.

[5] Blech, C., Appel, C. K., Ewert, R., Delfs, J. W., and Langer, S. C., "Numerical prediction of passenger cabin noise due to jet noise by an ultra-high-bypass ratio engine," Journal of Sound and Vibration, Vol. 464, 2020, p. 114960.

[6] Alder, M., Moerland, E., Jepsen, J., and Nagel, B., "Recent Advances in Establishing a Common Language for Aircraft Design with CPACS," Aerospace Europe Conference 2020, 2020. URL https://elib.dlr.de/134341/

[7] Wandel, M., "Design requirements of acoustic flight lab platform," INTER-NOISE and NOISE-CON Congress and Conference Proceedings, Vol. 253, Institute of Noise Control Engineering, 2016, pp. 3403-3412.

[8] Wandel, M., Grund, V., and Biedermann, J., "Validierung eines vibro-akustischen Simulationsmodells einer Flugzeugstruktur im mittleren Frequenzbereich,” Deutscher Luft- und Raumfahrtkongress, 4.-6. September 2018, Friedrichshafen, 2018. 
[9] Walther, J.-N., Kocacan, B., Hesse, C., Gindorf, A., and Nagel, B., "Automatisierte Kabinenvirtualisierung auf Basis von Flugzeugvorentwurfsdaten,” DLRK 2020, 2020. URL https://elib.dlr.de/135956/

[10] Lambe, A. B., and Martins, J. R., "Extensions to the design structure matrix for the description of multidisciplinary design, analysis, and optimization processes," Structural and Multidisciplinary Optimization, Vol. 46, No. 2, 2012, pp. $273-284$.

[11] Biedermann, J., Winter, R., Norambuena, M., Böswald, M., and Wandel, M., "Classification of the mid-frequency range based on spatial Fourier decomposition of operational deflection shapes," 24th International Congress on Sound and Vibration, 2017.

[12] Scherer, J., and Kohlgrüber, D., "Fuselage structures within the CPACS data format," Aircraft Engineering and Aerospace Technology: An International Journal, 2016.

[13] Siggel, M., Kleinert, J., Stollenwerk, T., and Maierl, R., “TiGL: An Open Source Computational Geometry Library for Parametric Aircraft Design,” Mathematics in Computer Science, 2019. URL https://elib.dlr.de/124524/

[14] Walther, J.-N., Hesse, C., Biedermann, J., and Nagel, B., "High Fidelity Digital Cabin Mock-Up based on Preliminary Aircraft Design Data for Virtual Reality Applications and Beyond," AIAA AVIATION Forum, 2021.

[15] Langer, P., Maeder, M., Guist, C., Krause, M., and Marburg, S., "More than six elements per wavelength: the practical use of structural finite element models and their accuracy in comparison with experimental results," Journal of Computational Acoustics, Vol. 25, No. 04, 2017, p. 1750025.

[16] VA One, “User's Guide 2020.1 (ESI Group)," 2020.

[17] Walther, J.-N., Petsch, M., and Kohlgrüber, D., "New approaches in CPACS-based preliminary design of aircraft fuselage structures using Python," 6th EASN International Conference, 2016. URL https://elib.dlr.de/107844/

[18] Winter, R., Biedermann, J., and Norambuena, M., "High-Resolution Vibration Measurement And Analysis Of The FlightLAB Aircraft Fuselage Demonstrator," INTER-NOISE 2018 - 47th International Congress and Exposition on Noise Control Engineering, 2018. URL https://elib.dlr.de/122597/

[19] Ansys, Structural Analysis Guide, Ansys Inc., Release 2020 R1 ed., 2020.

[20] Society of Automotive Engineers, AIR 1168/3: Aerothermodynamic systems engineering and design, SAE International, 1990. https://doi.org/https://doi.org/10.4271/AIR1168/3

[21] Kaszynski, A., "pyansys: Python Interface to MAPDL and Associated Binary and ASCII Files,” , Aug. 2020. https: //doi.org/\{10.5281/zenodo.4009467\}, URL https://doi.org/10.5281/zenodo.4009467

[22] Beckert, A., and Wendland, H., "Multivariate interpolation for fluid-structure-interaction problems using radial basis functions," Aerospace Science and Technology, Vol. 5, No. 2, 2001, pp. 125-134. https://doi.org/10.1016/s1270-9638(00)01087-7

[23] Quaranta, G., Masarati, P., and Mantegazza, P., "A conservative mesh-free approach for fluid structure problems," Int. Conf. on Computational Methods for Coupled Problems in Science and Engineering, 2005.

[24] Walther, J.-N., Gastaldi, A.-A., Maierl, R., Jungo, A., and Zhang, M., "Integration aspects of the collaborative aero-structural design of an unmanned aerial vehicle,” CEAS Aeronautical Journal, Vol. 11, No. 1, 2020, pp. 217-227.

[25] Krishnamurthy, T., "Comparison of Response Surface Construction Methods for Derivative Estimation Using Moving Least Squares, Kriging and Radial Basis Functions,” 2005. https://doi.org/10.2514/6.2005-1821.

[26] Geuzaine, C., and Remacle, J.-F., "Gmsh: A 3-D Finite Element Mesh Generator with Built-in Pre- and Post-Processing Facilities," International Journal for Numerical Methods in Engineering, Vol. 79, 2009, pp. 1309 - 1331. https://doi.org/10.1002/nme.2579

[27] Fuchs, M., Beckert, F., Biedermann, J., and Nagel, B., "Experience of Conceptual Designs and System Interactions for the Aircraft Cabin in Virtual Reality," AIAA Aviation Forum, 2021.

[28] Srinivasan, V., Markusheska, N., Walther, J.-N., Gindorf, A., Hesse, C., Biedermann, J., Meller, F., and Nagel, B., "Autonomous control of an Industrial robot based on formalized process description for cabin assembly," Deutsche Luft- und Raumfahrtkongress 2020, edited by V. Srinivasan, 2020. URL https://elib.dlr.de/136122// 